\title{
Microstructure simulation and mechanical properties of aisi1050 disk during quenching process
}

\author{
Jia Fu' ${ }^{1}$, a , Fuguo $\mathrm{Li}^{1}$, Yongtang $\mathrm{Li}^{3}$, Keqiang Wang ${ }^{2}$ and Huiping Qi ${ }^{3}$ \\ ${ }^{1}$ State Key Laboratory of solidification processing, School of Materials Science and Engineering, Northwestern \\ Polytechnical University, Xi'an 710072, China \\ ${ }^{2}$ Department of Material Chemistry, Southern University of Science and Technology, Shenzhen 518055, China \\ ${ }^{3}$ Materials Science and Engineering, TaiYuan University of Science \& Technology, Taiyuan 030024, China
}

\begin{abstract}
Microstructures of AISI1050 disk after quenching process (cooling from $880^{\circ} \mathrm{C}$ to $150^{\circ} \mathrm{C}$ by $6 \%$ AQ251 quenchant) were simulated by DEFORM-HT software, and the corresponding mechanical properties were calculated by the finite difference method (FDM). Above all, thermo-physical parameters and diagrams of CCT curve were calculated by JMATPRO software, and then the characterization of phase transformation and CCT curve were fatherly analyzed. Besides, the simulation of multi-phase microstructure considering initial thermo-physical parameters was realized by DEFORM-HT. The FDM method helps to obtain the volume fraction of single phase, and a weight function considering carbon equivalent of phases was introduced to predict the final hardness of multi-phase and quenched microstructure according to the single phase hardness simulated. This helps to explain the relationship between final hardness and various single phases.
\end{abstract}

Keywords: AISI1050 steel; numerical simulation; quenching; microstructure; hardness.

\section{Introduction}

During the quenching process, a series of phenomena exist, such as: heat transfer, phase transformation and mechanics change. Microstructure can be seen as temperature's derivatives [1], thus the quantitative analysis of quenching process becomes possible. Mechanical properties are inseparable to both microstructure and temperature. Research of quenching microstructure nowadays is mainly concentrated in metallographic observation, heat-transfer coefficient [2-3], phase-field model [4], martensitic transformations theory [5], temperature field calculation [6], TTT/CCT diagrams simulation [7] and the phase-field simulation [8] etc.. As material properties are relative with the microstructure distribution and the single-phase hardness is a certain value for the single phase microstructure, the hardness value of multiple-phase microstructures can be approximately predicted based on volume fractions of single phases. For volume fractions of single phases during quenching process, the changes of temperature field accompanied with phase transformation is considered.

The time-temperature-transformation (TTT) curve and continuous cooling transformation (CCT) curve can reflect the phase transformation characterization and provide evidence for quenching process. JMATPRO as a powerful software is commonly used in calculating TTT/CCT diagrams and

${ }^{a}$ Corresponding author : fuj@sustc.edu.cn 
thermo-physical properties [9, 10], which is commonly used to investigate properties of carbon steel, the low alloy steels, Cr-Mo steels [11] and multi-component alloys [12] and nickel-based super alloys [13]. It can also be enlarged to investigate high temperature deformation behavior [14], to establish the phase - field model [4] and to simulate the microstructure [16]. During cooling process at a constant rate, phase transformations occurred as well as performance parameters of different phases formed during quenching process. The theoretical analysis [17], microstructure simulation [17] and the experiment [16] of as-cast 42CrMo steele have been investigated, which helps us to investigate the medium-carbon alloy steel -AISI1050 steel. AISI1050 steel is mainly used to produce the train wheel, gear, friction disk and mandrel with small cross-sections [18]. Sunay [19] has studied microstructures and mechanical properties of AISI1050 steel during quenching process with a higher wear resistance under complicated load condition. This work aims to get the relationship between multiple-phase hardness and single-phase hardness, and then provide the quenched hardness model of AISI1050 steel.

\section{Material model and thermo-physical parameters}

\subsection{Material phase diagram and TTT curve}

Element chemical composition(wt\%) result of AISI1050 steel is as follows: C: $0.48 \%$, Si: $0.22 \%$, Mn: $0.8 \%$, P:0.02\%, Cr: $0.18 \%$, Mo: $0.23 \%$, S: $0.02 \%$, Cu: $0.18 \%$, others Fe $97.87 \%$. Considering the practical processing of AISI1050 steel ${ }^{[20]}$, the fully austenitizing temperature is set at $860^{\circ} \mathrm{C}$. The element chemical composition is input first, and the alloy phase equilibrium diagram and TTT curve by JMATPRO software is obtained, separately shown in Fig.1 (a) and Fig.1 (b). Properties
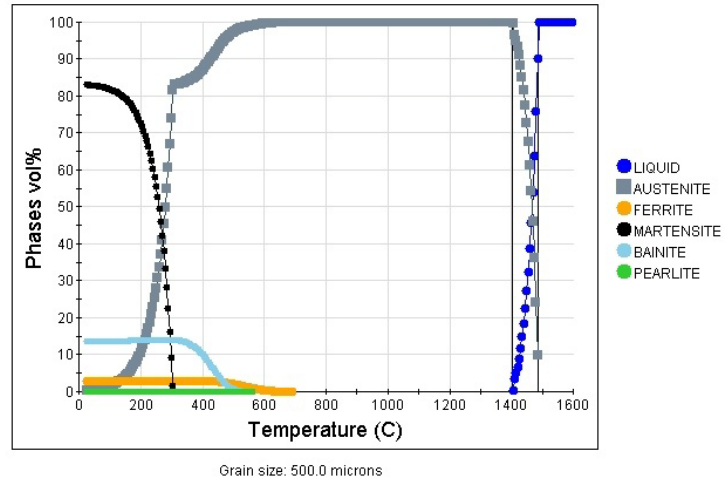

b)

TTT

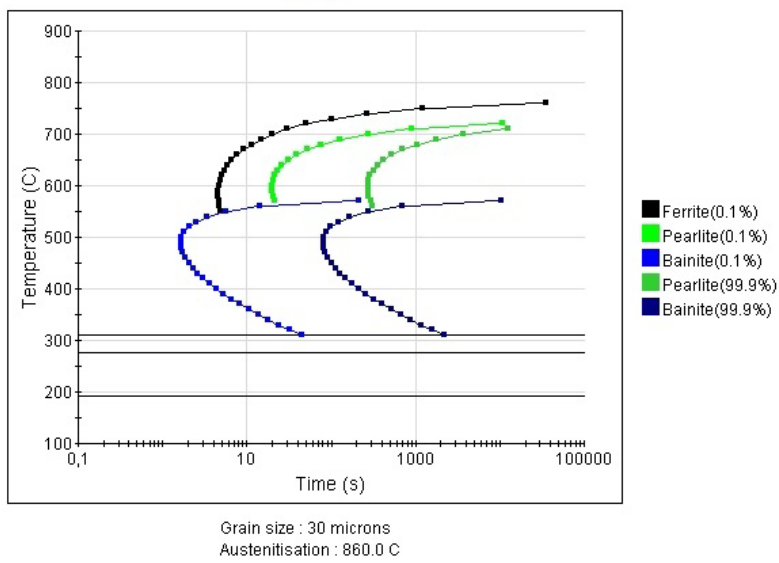

Figure 1. Calculation curves: (a) Calculation of phase equilibrium; (b) TTT curve; 
Fig.1 (a) has shown phase areas and critical temperature points, in which phase equilibrium zones mainly include liquid zone, ferrite zone, austenite zone and cementite zone, and others are alloy carbides zones, suchas $\mathrm{M}_{23} \mathrm{C}_{6}, \mathrm{M}_{2} \mathrm{P}, \mathrm{M}_{7} \mathrm{C}_{3}, \mathrm{MnS}$ and $\mathrm{M}_{2}(\mathrm{C}, \mathrm{N})$. Besides, austenitic temperature region is $769.5^{\circ} \mathrm{C} \sim 880^{\circ} \mathrm{C}$, martensite transformation region is $156^{\circ} \mathrm{C} \sim 311^{\circ} \mathrm{C}$, bainite transformation region is $317^{\circ} \mathrm{C} \sim 573^{\circ} \mathrm{C}$ and pearlite transformation region is $560^{\circ} \mathrm{C} \sim 727^{\circ} \mathrm{C}$. Fig. 1 (b) has shown the simulated TTT curve, of which the fully austenitizing temperature $\mathrm{Ac}_{3}$ is at $760^{\circ} \mathrm{C}$, martensitic starting temperature ( $M s$ )is at $310.9^{\circ} \mathrm{C}$ and martensitic finishing temperature $\left(M_{f}\right)$ is at $191.3^{\circ} \mathrm{C}$.

\subsection{CCT curve of AISI1050 during quenching process}

For AISI1050 steel, the simulated and practical CCT curves by reference [20] are in Fig.2.

a)

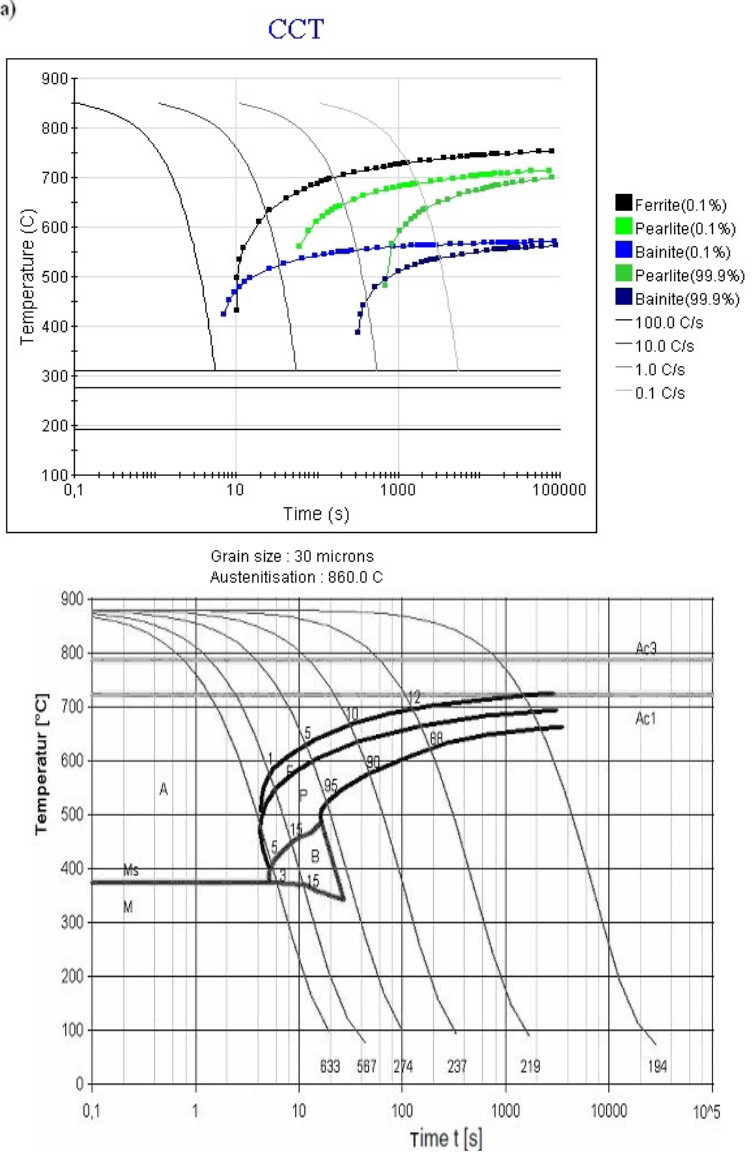

Figure 2. (a) Simulated CCT curve; (b) Practical CCT curve.

Fig.2 (a) has shown the simulated CCT curve, of which the cooling rate $V_{c}$ value is about $84^{\circ} \mathrm{C} / \mathrm{s}$. It is indicated from Fig.2 (b) that the cooling rate has greatly influenced the final microstructure after quenching. If we choose a kind of $6 \% \mathrm{AQ} 251$ quenchant (the averaged cooling rate $82.8^{\circ} \mathrm{C} / \mathrm{s}$ ), the theoretical microstructure inferred by CCT curve should be martensite and lower bainite, and mechanical properties after quenching with martensite and lower bainite will be greatly improved.

\subsection{Thermo-physical parameters and material model}

Thermo-physical properties parameters at room temperature of AISI1050 steel are shown as follows [21]: density $7860 \mathrm{~kg} / \mathrm{m}^{3}$, Yong's modulus 208GPa, Poisson's ratio 0.297, yield strength 365.4MPa, 
tensile strength $636 \mathrm{MPa}$, thermal expansion $11.65 \times 10^{-6} \mathrm{~K}^{-1}$, conductivity $49.4 \mathrm{~W} \cdot \mathrm{m}^{-1} \cdot \mathrm{k}^{-1}$, Specific heat $477 \mathrm{~J} \cdot \mathrm{kg}^{-1} \cdot \mathrm{K}^{-1}$. Other related thermo-physical parameters during various temperatures can be obtained by JMATPRO is shown in Table 1.

Table 1. Thermo-physical properties of AISI1050 steel

\begin{tabular}{lccccccc}
\hline Temperature ${ }^{\circ} \mathrm{C}$ & 200 & 400 & 600 & 700 & 800 & 900 & 1000 \\
\hline Density $\left(\mathrm{kg} / \mathrm{m}^{3}\right)$ & 7785 & 7730 & 7650 & 7645 & 7640 & 7585 & 7540 \\
Enthalpy $(\mathrm{kJ} / \mathrm{kg})$ & 91.00 & 200 & 375 & 506 & 570 & 610 & 675 \\
Specific heat $\left(\mathrm{kJ} \cdot \mathrm{kg}^{-1} \cdot \mathrm{K}^{-1}\right)$ & 0.51 & 0.60 & 0.76 & 1.05 & 0.61 & 0.62 & 0.65 \\
Conductivity $\left(\mathrm{W} \cdot \mathrm{m}^{-1} \cdot \mathrm{k}^{-1}\right)$ & 42.0 & 36.0 & 33.00 & 29.0 & 27.0 & 28.0 & 29.0 \\
\hline
\end{tabular}

Import these parameters above into the material window of DEFORM to establish the material model. Then quenching microstructure will be simulated on DEFORM based on the material model.

\section{Results and discussion}

\subsection{Disk model and boundary condition}

We set the initial conditions of simulation on DEFORM software as follows: environmental temperature is $20^{\circ} \mathrm{C}$, averaged thermal transfer coefficient 27 , quenching temperature $880^{\circ} \mathrm{C}$, time step $0.02 \mathrm{~s}$, step number 2500. Convective heat is the main consideration during quenching, and quenching harden ability data simulated by JMATPRO is obtained and then imported into the Jominy window of material for the preparation of microstructure simulation by DEFORM.. Latent heat of phase transformation of ferrite $/$ pearlite, bainite and martensite during simulation are $6.02 \times 10^{8} \mathrm{~J} / \mathrm{m}^{3}$, $4.40 \times 10^{8} \mathrm{~J} / \mathrm{m}^{3}$ and $6.28 \times 10^{8} \mathrm{~J} / \mathrm{m}^{3}$ respectively [22]. Sub-hexahedral element is selected on DEFORMHT and the element number is chosen as 40,000 with grid number divided. Intensively mesh the nearby surface of disk and add material model and phase transformation models (Demo_Temper_Steel.KEY files), the simulated temperature field, hot stress field, microstructure distribution and hardened layer distribution are finally obtained and displayed.

\subsection{Microstructure and hardness distribution}

Simulated temperature field distribution, temperature curve during heating, stress field distribution and hot-stress curve of a quarter disk model during quenching process are shown in Fig.3.
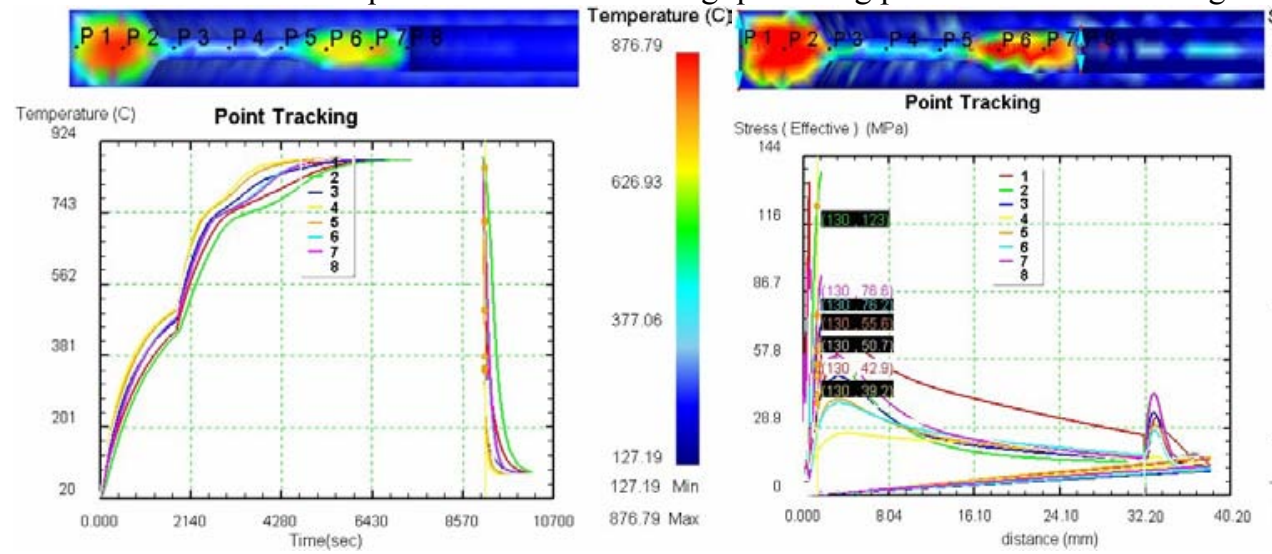

Stress - Mean (MPa:

Figure 3. Simulation curves of a quarter-disk: (a) Temperature field; (b) hot-stress field

As is shown in Fig.3(a), temperature gradient appears during the period of heating to $880^{\circ} \mathrm{C}$, which can be interpreted as that AISI1050 steel has a small thermal conductivity $\left(27.5 \mathrm{~W} \cdot \mathrm{m}^{-1} \cdot \mathrm{k}^{-1}\right.$ or so). After 
temperature has reached the fully austenitized temperature, the temperature gradient is gradually eliminated accompanied with prolonging holding time. It is also indicated that a series of discontinuous inflection appear in temperature curve, and it is because the discontinuous element during finite element calculation is by the thermal conductivity and thermal conductivity is the function of temperature. The hot stress field is shown in Fig.3 (b), due to thermal expansion and cold contraction as well as uneven thermal diffusion, surface turns out to be tensile stress (negative) and center is compressed stress (positive). The mean stress difference between maximum stress (-62.3MPa) and minimum stress $(33.5 \mathrm{MPa})$ is up to $95.8 \mathrm{MPa}$, which needs the further tempering process to eliminate it. From Fig.3 (b) we can also see that the tensile stress on the top nearby is so large that stress concentration is easy to start up. If these areas stress in usage is over than threshold value of crack spreading $\Delta K_{\text {th }}$ [23-24], micro-cracks will appear and cracks may be enlarged thus led to failure.

\subsection{Microstructure simulation}

The simulated microstructure and hardness after quenching at step 2200 are shown in Fig.4.

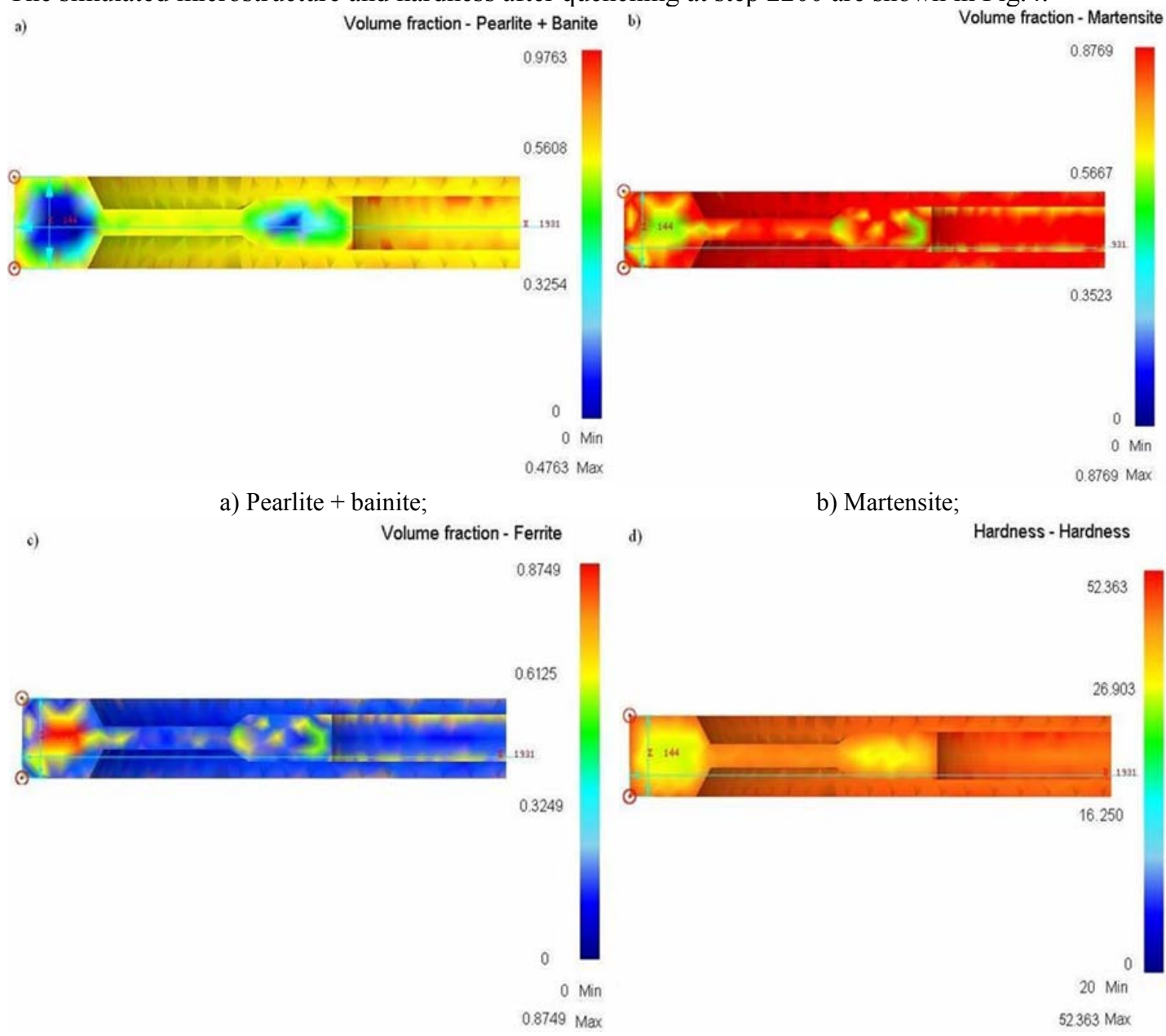

c) Ferrite;

d) Hardness

Figure 4. Simulated microstructure and hardness

From Fig.4, quenching at $880^{\circ} \mathrm{C}$ in $6 \% \mathrm{AQ} 251$, the surface is mixed microstructure of bainitemartensite in main, while the center is mixed ferrite-pearlite. Phase volume fractions of various phase transformations with different distance from the left end are observed in Fig.3 and shown in Tab.2. 
Based on these volume fractions of ferrite/pearlite, bainite and martensite, the theoretical hardness of multiple phases can be calculated. The surface hardness is about 52.4HRC on average and the central partis about27HRC, which is approachable to the surface hardness $54.8 \mathrm{HRC}$ and center hardness 28.2 HRC calculated by FDM.

Table 2. Phase volume fractions

\begin{tabular}{ccccccc}
\hline \multicolumn{2}{c}{$\begin{array}{c}\text { Distance from } \\
\text { surface }\end{array}$} & $0 \mathrm{~mm}$ & $10 \mathrm{~mm}$ & $20 \mathrm{~mm}$ & $25 \mathrm{~mm}$ & $40 \mathrm{~mm}$ \\
\hline Phase & $\mathrm{F}+\mathrm{P}$ & 0 & 0 & 0.008 & 1.126 & 4.923 \\
Fractions & $\mathrm{B}$ & 0 & 1.126 & 36.301 & 47.611 & 77.425 \\
$(\%)$ & $\mathrm{M}$ & 100 & 98.874 & 63.691 & 51.263 & 17.652 \\
\hline
\end{tabular}

In all, based on the assumption that hardness is the function of phase hardness and its volume fraction, the high-temperature thermal physical properties is obtained by the JMATPRO database, then the material model is established to simulate the microstructure on DEFORM-HT, and then the weight function considering carbon equivalent of phases was introduced and added to calculate the hardness based on the simulated microstructure.

\section{Compasion of experiment and simulation}

\subsection{Experimental procedure}

AISI1050 steel sample is chosen as $\Phi 100 \mathrm{~mm} \times 100 \mathrm{~mm}$, which is over the critical size $\left(D_{C} \approx 45 \mathrm{~mm}\right)$. The quenching process is as follows: heating to $880^{\circ} \mathrm{C}$ and holding about $90 \mathrm{~min}$, then quenching in the $6 \%$ AQ251 quenching medium to $150^{\circ} \mathrm{C}$. Heating process is as follows: heating to $700^{\circ} \mathrm{C}$ with a holding time for $1 \mathrm{~h}$ to eliminate the temperature gradient, and then heating to 880 with a holding time for $2.5 h$ to be fully austenitized by SRJX-4-13 box-type furnace. To observe microstructure on various positions of sample, we have cut the central part of samples and made it polished, and then the polished sample is corroded within a 4\% nitric acid alcohol. Various microstructure regions are observed by VEX-600E metallographic microscope and Rockwell hardness is repeatedly measured on HR-150A Rockwell tester to get an average value.

\subsection{Metallographic analysis and hardness analysis}

Microstructure of AISI1050 observed by VEX-600E metallographic microscope is in Fig.5.
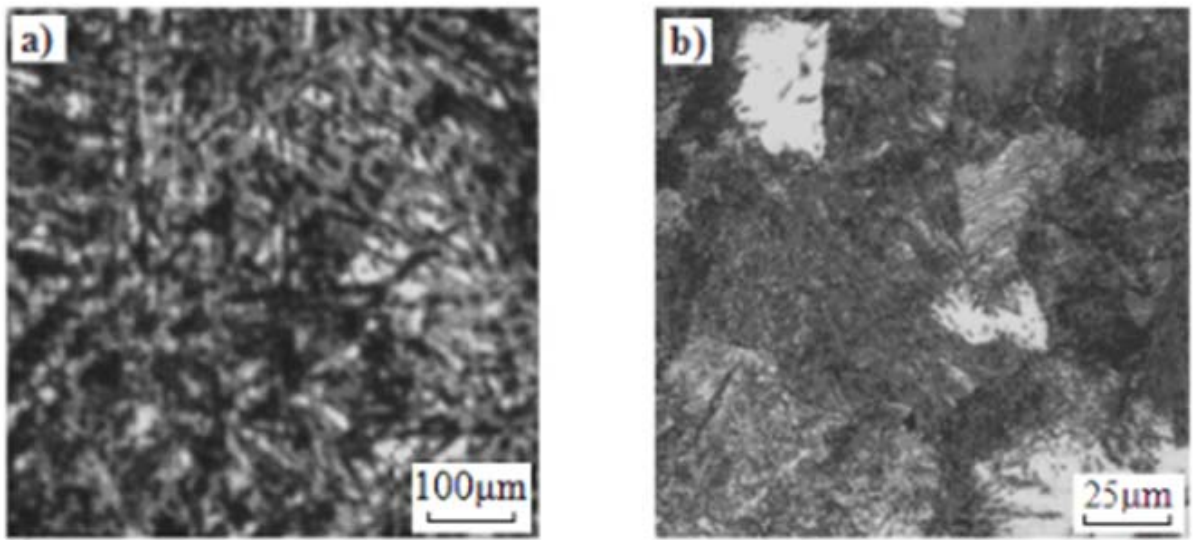

Figure 5. Quenching microstructures: (a) Surface microstructure $(0 \mathrm{~mm})$; (b) Centre microstructure $(25 \mathrm{~mm})$ 
As is shown in Fig. 5(a), surface microstructure is mainly composed of cryptocrystalline martensite, lath martensite and lower bainite, with slice retained austenite between laths. Quenching from fully austenitizated temperature in the 6\% AQ251 quenching medium has caused the retained austenite distributed as films between the martensite laths. The thermal stabilization of austenite is accompanied with the process of carbon partitioning, and phase transformation from martensite to austenite occurs across low-mobility martensite-austenite interfaces [25]. The K-S orientation relationship is retained by rapid quenching. High density of dislocations can be observed inside lath martensite, which can move at greater ease and relief the local stress concentration of crack nucleation part, and which can also reduce the peak stress value of crack tip thus is good for toughness. While the centre microstructure is mainly constituted of pearlite(dark areas) and ferrite(light areas) [26], with slatted pearlite as matrix and some Mn carbides dispersed observed in Fig. 5(b).

\subsection{Comparison of simulation and experiment}

Fig. 5 shows the average hardness comparison of calculation and experiment. Within the distance range of 5-45mm, the experimental hardness measured by HR-150A Rockwell tester is in Fig.6.

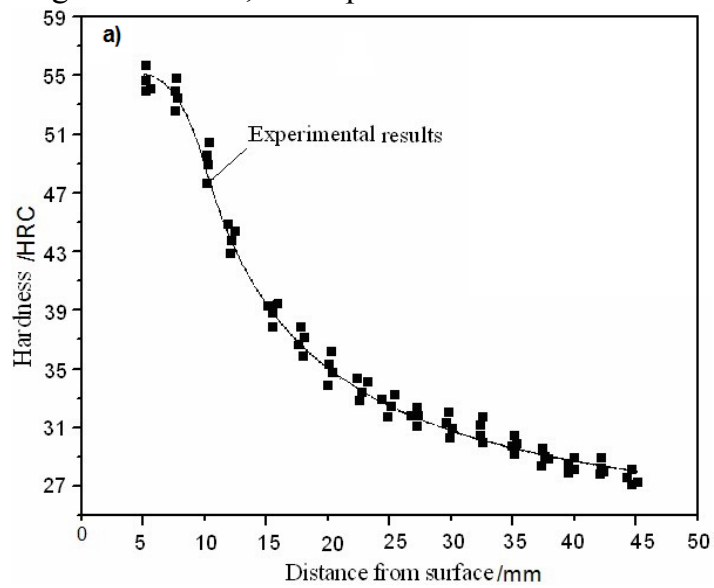

a) Four-order polynomial fit of experiment data.

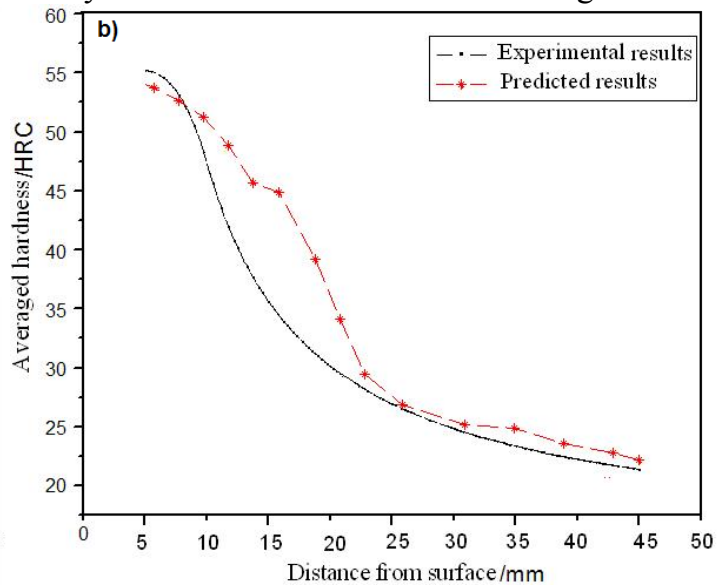

b) Hardness comparison of experiment and simulation.

Figure 6. Comparison of experiment and simulated hardness.

As is shown in Fig.6, measured average hardness on surface is 55HRC and in center 28HRC. The hardness formula can be fitted by five order polynomial based on the experimental data as:

$$
H_{x}=55.537-6.133 \times 10^{-5} \cdot x^{4}+6.440 \times 10^{-3} \cdot x^{3}-0.208 x^{2}+1.359 x
$$

As martensite makes the most contribution to hardness, the phase transformation model of martensite was fatherly investigated.

With comparison of hardness, the relative error between measured average hardness and hardness simulated is only $4.72 \%$ on surface and $3.57 \%$ in centre, indicating that numerical simulation to predict the hardness has certain guidance. It is also indicated that the method by introducing weight function to explain mapping relations between simulated hardness and simulated microstructures during quenching process has a certain credibility and feasibility.

As the experiment data has a good agreement with the calculated values within distance $25 \mathrm{~mm}$ $40 \mathrm{~mm}$, simulated hardness can provide some basis to predict the hardness on certain degree. The FDM algorithm is a good way to investigate the nonlinear problems of temperature field, microstructure transformation, thermo-mechanical coupling and phase transformation during quenching where the heat enthalpy can not be ignored, and it has a certain value in engineering application.

The proposed method has provides some base to the hardness prediction and mechanical analysis for other quenched steel. So it is can be anticipated that the analyzing of microstructure simulation and 
properties prediction demonstrated in this work could be expanded to other properties research and material design fields where phase transformations occur.

\section{Conclusion}

Assuming that hardness is the function of phases, the proposed method to calculate hardness distribution of AISI1050 steel during quenching process can be realized to predict hardness distribution by combining JMATPRO and DEFORM-HT. Based on the thermo-physical parameters calculated by JMATPRO, quenching process (cooling from $880^{\circ} \mathrm{C}$ to $150^{\circ} \mathrm{C}$ by $6 \% \mathrm{AQ} 251$ quenchant ) is simulated on DEFORM-HT software. Results are as:

1) Metallographic observation and hardness experiment were carried out. Simulated microstructures are mainly martensite + bainite on surface and pearlite + ferrite in centre, which is roughly in accord with the experimental microstructure. The experimental microstructure includes martensite + lower bainite + little retained austenite on surface and pearlite-type + ferrite in centre.

2) Based on phase volume fractions by using FDM and weight values considering carbon equivalent of phases, hardness model is proposed. By hardness comparison, we can find that hardness of simulated microstructure is $54.8 \mathrm{HRC}$ on surface and $28.2 \mathrm{HRC}$ in centre, which is close to the experimental average value, 55HRC on surface with $28 \mathrm{HRC}$ at center. Besides, the relative error is only $4.72 \%$ on surface and $3.57 \%$ at centre and thus is acceptable.

3) By comparing hardness curves obtained by predicated model and experimental fitting, it shows that the calculated values using FDM method have a good tendency with the experimental data within distance $25 \mathrm{~mm}-40 \mathrm{~mm}$, thus the model based on weights and simulated microstructures is basically verified.

4) It can be concluded that hardness of quenched AISI1050 steel can be predicted by the method proposed, which helps to understand relationship between hardness and microstructures.

In all, the theoretical calculation result is close to the simulated and experimental ones and the relative error is under an acceptable region, thus the modified model for predicting hardness distribution based on weight coefficients and microstructures simulated is verified. These researches above are of great reference and significance to the practical production.

\section{Acknowledgements}

This work is supported by the National Science Foundation (51075290, a grant from the Aeronautical Science Foundation of China (2011ZE53059) and the financial support by China Scholarship Council (CSC).

\section{References}

1. Y. Kimura, Tadanobu Inoue, Fuxing Yin, Kaneaki Tsuzaki, Science, 320,5879, 1057-1060 (2008) .

2. Z. Li, G. Ramana, and R. Shivpuri, Int. J. Machine Tools Manufact., 42, 549-558 (2002).

3. Z. Li, G. Ramana, and R. Srinivasan, J. Mater. Proc. Technol., 172(2), 249-257 (2006).

4. I. Kovačević, Božidar Šarler, Mater. Sci. Eng.A, 413-414,423-428 (2005).

5. K. Bhattacharya, S. Conti, G. Zanzotto, J. Zimmer, Nature, 428(6978),55-59 (2004).

6. H. Cheng, T. He and J. Xie, J. Mater. Sci. Technol., 4, 372-374 (2002).

7. Z.Guo, N.Saunders, A.P. Miodownik, J.-Ph. Schillé, Mater. Sci. Eng.A, 413-414, 465-469 (2005).

8. X. Yu, Fuguo Li, Y. Ren, et al., J. Mater. Sci. Technol., 22(4), 441-446 (2006).

9. JP. Schille, ZL. Guo, N. Saunders, AP. Miodownik, J. Mater Manuf Process, 26(1), 137-143 (2011).

10. H.T. Lee, CT. Chen and JL. Wu, J. Mater. Proc. Technol., 210( 12), 1636-1645 (2010). 
11. S. Hatami, A. Malakizadi, L. Nyborg, D.Wallin, J. Mater. Proc. Technol., 210 ( 9), 1180-1189 (2010).

12. I.Kovačević, Mater. Sci. Eng.A, 496 (1-2),345-354 (2008).

13. Z. Guo, N. Saunders, JP. Schille, AP. Miodownik, Mater. Sci. Eng.A, 499 (1-2), 7-13 (2009).

14. Y. Li, H. Qi, Q. Li et al.. Journal of Mechanical Engineering, 49(20), 49-54. (2013)

15. LI Yongtang, YANG Qing,QI Huiping, et al..China Mechanical Engineering, 50(16): 77-82. (2014)

16. H. Qi, Y. Li, J. Fu, Z. Liu, Advanced Materials Research, 314- 316, 2560-2564 (2012).

17. J. Fu, Y. Li and H. Qi, Advanced Materials Research, 317-319, 19-23 (2011).

18. N.Y. Sari and M. Yilmaz, J. Mater. Des., 27(6), 470-478 (2006).

19. T. Y. Sunay,M. Sahin and S. Altintas, Int. J. Adv. Manuf. Technol., 44, 68-79 (2009).

20. J. Ohrlich, H. J. Pietrzeniuk, Atlas zur W"armebehandlung der St" ahle, Bd. 4, Stahleisen, 1976.

21. S.H.Tang, Y.M.Kong, S.M.Sapuan, et al., J. Mater. Proc. Technol., 171( 2),259-267 (2006).

22. S. Denis, D. Farias and A. Simon, ISIJ Int., 323, 316-325(1992).

23. J.T. Maximov, A.P. Anchev, Int. J. Machine Tools Manufact., 43(12) ,1241-1251 (2003).

24. X. Zhang, Z. Wang, Int. J. Fatigue. vol. 25(9-11), 1249-1257 (2003).

25. M. J. Santofimia, L. Zhao, R. Petrov, et al., Acta. Mater., 59(15),6059-6068 (2011).

26. S.F Luk, T.P Leung, W.S Miu and I Pashby, J. Mater. Proc. Technol., 91 (1-3), 245-249 (1999). 